\title{
Ultra-Wideband Time-Difference-Of-Arrival High Resolution 3D Proximity Tracking System
}

\author{
Jianjun Ni* , Dickey Arndt+, Phong Ngo+, Chau Phan+, Kent Dekome+, John Dusl\# \\ *ERC/ESCG, ${ }^{+}$NASA Johnson Space Center, \#Jacobs/ESCG \\ *david.ni-1@nasa.gov
}

\section{BIOGRAPHY}

Jianjun (David) Ni received his Ph.D. degree in Electrical Engineering from Iowa State University in 2002. From 2001 to 2003, he was a research staff in Department of Electrical \& Computer Engineering at University of Houston. Dr. Ni received the National Research Council (NRC) Research Associateship Award and joined NASA Johnson Space Center (JSC) as a NRC research associate in 2003. In 2005, he joined ERC/ESCG to support the Orion Communication and Tracking (C\&T) System analysis work at JSC/NASA. Dr. Ni's research interests fall in the general areas of communications and tracking, signal processing and information theory.

\section{ABSTRACT}

This paper describes a research and development effort for a prototype ultra-wideband (UWB) tracking system that is currently under development at NASA Johnson Space Center (JSC). The system is being studied for use in tracking of lunar/Mars rovers and astronauts during early exploration missions when satellite navigation systems are not available. UWB impulse radio (UWB-IR) technology is exploited in the design and implementation of the prototype location and tracking system. A threedimensional (3D) proximity tracking prototype design using commercially available UWB products is proposed to implement the Time-Difference- Of-Arrival (TDOA) tracking methodology in this research effort. The TDOA tracking algorithm is utilized for location estimation in the prototype system, not only to exploit the precise time resolution possible with UWB signals, but also to eliminate the need for synchronization between the transmitter and the receiver. Simulations show that the TDOA algorithm can achieve the fine tracking resolution with low noise TDOA estimates for close-in tracking. Field tests demonstrated that this prototype UWB TDOA High Resolution 3D Proximity Tracking System is feasible for providing positioning-awareness information in a 3D space to a robotic control system. This 3D tracking system is developed for a robotic control system in a facility called "Moonyard" at Honeywell Defense \& System in Arizona under a Space Act Agreement.

\section{INTRODUCTION}

A few prototype ultra-wideband (UWB) tracking systems are currently under development at NASA Johnson Space Center (JSC). These systems are being studied for use in tracking of Lunar/Mars rovers and astronauts during early exploration missions when satellite navigation systems (such as GPS) are not available. UWB technology is exploited to implement the tracking system due to its fine time resolution, which is on the order of picoseconds, its low power spectral density, which allows the system to coexist with other communication systems, and its resistance to multipath interference. The tracking algorithms such as Time-Difference-Of-Arrival (TDOA) and Angle-Of-Arrival (AOA) are utilized by these systems for various space applications, such as long range tracking, proximity high resolution tracking, and trackingdata-aided docking. Some analysis and design efforts have been made to demonstrate the feasibility of UWB tracking systems and improve the tracking accuracy [1-8]. In this paper, we document a research effort that extends the tracking capability of the UWB TDOA proximity high resolution tracking system from a two-dimensional (2D) space to a three-dimensional (3D) space. This 3D tracking system is developed for a robotic control system in a facility called "Moonyard" at Honeywell Defense \& System in Arizona under a Space Act Agreement.

The remainder of this paper is organized as follows. In Section II, the TDOA tracking methodology is introduced and the $3 \mathrm{D}$ tracking algorithm is derived. The simulation results are discussed in Section III. In Section VI, prototype system design and filed tests are discussed. Some concluding remarks are presented in Section VI.

\section{TRACKING METHODOLOGY ALGORITHM}

AND

\section{A. TDOA Methodology}

Many methods have been applied to estimate the location of a radio source, such as angle of arrival (AOA), time difference of arrival (TDOA), relative signal strength (RSS) and various hybrids of them. For close-in applications, the TDOA approach has been chosen as the 
tracking method in this research effort since it does not require synchronization between the transmitter and receiver. Furthermore, the extremely high fidelity of the UWB timing circuitry permits very high accuracy of propagation time measurements while transmitting data. This fine time resolution feature of UWB can provide the accurate TDOA estimates required for reliable tracking system performance. The TDOA technique is discussed briefly below.

Since electromagnetic waves travel with constant velocity in free space, the distance between the transmitter and the receiver is directly proportional to the propagation time of the signal. The TDOA approach determines the possible position of the transmitter by examining the difference in time at which the same signal arrives at multiple receivers. Each TDOA measurement determines that the transmitter must lie on a hyperboloid with constant range difference between the two receivers. At least three receivers are needed for a $2 \mathrm{D}$ location estimation, and four receivers for a 3D location estimation. The intersection of hyperboloids provides the location of the transmitter.

Generally, the transmitter location is determined by finding a least-squares (LS) solution to linearized versions of the TDOA equations. The conventional method for linearization uses Taylor series expansion, which has two major drawbacks: the requirement of a good initial guess and no guarantee of convergence. A Two-Stage Weighted Least Square Solution (TSWLSS) algorithm can eliminate the initialization and convergence problems [9]. This method linearizes the TDOA equations by adding a dummy variable. The original non-linear TDOA equations are transformed into a set of linear equations with an extra variable which requires one more receiver or sensor. Since this algorithm nearly achieves the Cramer-Rao lower bound for Gaussian TDOA noise at low noise levels, it corresponds to a nearly optimal estimator for this problem [10].

\section{B. 3D Tracking Algorithm}

The $3 \mathrm{D}$ version of the TSWLS algorithm is derived as follows. Assume that there is one transmitter located at an unknown location $(x, y, z)$ in three-dimensional space and five receivers located at positions $\left\{(0,0,0),\left(x_{2}, y_{2}, z_{2}\right),\left(x_{3}, y_{3}, z_{3}\right),\left(x_{4}, y_{4}, z_{4}\right),\left(x_{5}, y_{5}, z_{5}\right)\right\}$, which are assumed to be known precisely. Further, assume that measurements of the relative time delays $\left\{\tau_{21}, \tau_{31}, \tau_{41}, \tau_{51}\right\}$ between the arrival of the transmitted signal at receiver $(0,0,0)$ and each of the other locations $\left(x_{2}, y_{2}, z_{2}\right), \ldots,\left(x_{5}, y_{5}, z_{5}\right)$ are available.
If the propagation velocity of the signals is given by the constant $c$, then it can be shown that the following system of linear equations is satisfied:

where

$$
\mathbf{G}_{1} \mathbf{P}_{1}=\mathbf{h}_{1}
$$

$$
\begin{gathered}
\mathbf{G}_{1}=2\left[\begin{array}{cccc}
x_{2} & y_{2} & z_{2} & c \tau_{21} \\
x_{3} & y_{3} & z_{3} & c \tau_{31} \\
x_{4} & y_{4} & z_{4} & c \tau_{41} \\
x_{5} & y_{5} & z_{5} & c \tau_{51}
\end{array}\right], \mathbf{P}_{1}=\left[\begin{array}{l}
x \\
y \\
z \\
d_{1}
\end{array}\right], \\
\mathbf{h}_{1}=\left[\begin{array}{l}
x_{2}^{2}+y_{2}{ }^{2}+z_{2}^{2}-\left(c \tau_{21}\right)^{2} \\
x_{3}^{2}+y_{3}{ }^{2}+z_{3}^{2}-\left(c \tau_{31}\right)^{2} \\
x_{4}{ }^{2}+y_{4}{ }^{2}+z_{4}{ }^{2}-\left(c \tau_{41}\right)^{2} \\
x_{5}{ }^{2}+y_{5}{ }^{2}+z_{5}{ }^{2}-\left(c \tau_{51}\right)^{2}
\end{array}\right],
\end{gathered}
$$

and $d_{1}$ can be treated as a dummy variable for $d_{1}^{2}=x^{2}+y^{2}+z^{2}$.

The TDOA measurements noise is assumed to be a zeromean Gaussian random vector with covariance matrix $\mathbf{Q}$ such that

$$
\overline{\Delta \tau} \in \eta(\mathbf{0}, \mathbf{Q})
$$

and

$$
\mathbf{Q}=\left[\begin{array}{cccc}
\sigma^{2} & 0 & 0 & 0 \\
0 & \sigma^{2} & 0 & 0 \\
0 & 0 & \sigma^{2} & 0 \\
0 & 0 & 0 & \sigma^{2}
\end{array}\right]
$$

\section{Stage I}

The error in the system corresponds to $\varepsilon_{1}=\mathbf{h}_{1}-\mathbf{G}_{1} \mathbf{P}_{1}$. The steps to solving the weighted least squares solution of $\mathbf{P}_{1}$ that minimizes $\varepsilon_{1}^{T} \mathbf{W}_{1} \varepsilon_{1}$ are shown next. First, a farfield source is assumed, thus the transmit position becomes:

$$
\mathbf{P}_{11}=\left(\mathbf{G}_{1}^{T} \mathbf{Q}^{-1} \mathbf{G}_{1}\right)^{-1} \mathbf{G}_{1}{ }^{T} \mathbf{Q}^{-1} \mathbf{h}_{1}
$$

For a near-field source, this estimation is further refined using the weighted LS solution shown below:

where

$$
\mathbf{P}_{1}=\left(\mathbf{G}_{1}^{T} \mathbf{W}_{1} \mathbf{G}_{1}\right)^{-1} \mathbf{G}_{1}^{T} \mathbf{W}_{1} \mathbf{h}_{1}
$$




$$
\mathbf{B}_{1}=\left[\begin{array}{cccc}
d_{2} & 0 & 0 & 0 \\
0 & d_{3} & 0 & 0 \\
0 & 0 & d_{4} & 0 \\
0 & 0 & 0 & d_{5}
\end{array}\right], \mathbf{W}_{1}=\frac{1}{4 c^{2}} \mathbf{B}_{1}{ }^{-1} \mathbf{Q}^{-1} \mathbf{B}_{1}^{-1}
$$

\section{Stage II}

In order to satisfy the equation $d_{1}^{2}=x^{2}+y^{2}+z^{2}$, the following equation must hold

$$
\mathbf{G}_{2} \mathbf{P}_{2}=\mathbf{h}_{2}
$$

where

$$
\mathbf{G}_{2}=\left[\begin{array}{lll}
1 & 0 & 0 \\
0 & 1 & 0 \\
0 & 0 & 1 \\
1 & 1 & 1
\end{array}\right], \mathbf{P}_{2}=\left[\begin{array}{c}
x^{2} \\
y^{2} \\
z^{2}
\end{array}\right] \text {, and } \mathbf{h}_{2}=\left[\begin{array}{l}
\mathbf{P}_{1}^{2}(1) \\
\mathbf{P}_{1}^{2}(2) \\
\mathbf{P}_{1}^{2}(3) \\
\mathbf{P}_{1}^{2}(4)
\end{array}\right]
$$

The error for this stage is equivalent to $\varepsilon_{2}=\mathbf{h}_{2}-\mathbf{G}_{2} \mathbf{P}_{2}$. The least squares solution of $\mathbf{P}_{2}$ which will minimizes $\varepsilon_{2}{ }^{T} \mathbf{W}_{2} \varepsilon_{2}$ will refine the location estimate from the previous stage. This least squares solution can be represented by the equation below:

where

$$
\mathbf{P}_{2}=\left(\mathbf{G}_{2}{ }^{T} \mathbf{W}_{2} \mathbf{G}_{2}\right)^{-1} \mathbf{G}_{2}{ }^{T} \mathbf{W}_{2} \mathbf{h}_{2}
$$

$$
\begin{gathered}
\mathbf{W}_{2}=\frac{1}{4} \mathbf{B}_{2}^{-1} \mathbf{G}_{1}^{T} \mathbf{W}_{1} \mathbf{G}_{1} \mathbf{B}_{2}{ }^{-1}, \\
\mathbf{B}_{2}=\left[\begin{array}{cccc}
\mathbf{P}_{1}(1) & 0 & 0 & 0 \\
0 & \mathbf{P}_{1}(2) & 0 & 0 \\
0 & 0 & \mathbf{P}_{1}(3) & 0 \\
0 & 0 & 0 & \mathbf{P}_{1}(4)
\end{array}\right] .
\end{gathered}
$$

\section{Final Solution}

The final estimate of the tracked location is given by the equation

$$
\mathbf{P}=\mathbf{S} \sqrt{\mathbf{P}_{2}}=\left[\begin{array}{c}
x \\
y \\
z
\end{array}\right]
$$

where

$$
\mathbf{S}=\left[\begin{array}{ccc}
\operatorname{sign}\left[\mathbf{P}_{1}(1)\right] & 0 & 0 \\
0 & \operatorname{sign}\left[\mathbf{P}_{1}(2)\right] & 0 \\
0 & 0 & \operatorname{sign}\left[\mathbf{P}_{1}(3)\right]
\end{array}\right]
$$

assuming that the sign of each coordinate in $\mathbf{P}_{1}$ is correct.

\section{TRACKING SIMULATIONS}

It is shown in [7] for the tracking application in a 2D space that given a receiver configuration, the tracking performance mean-squared-error (MSE) is a function of some critical parameters such as variance of TDOA estimates, the tracking range, and the radius of baseline (space for receiver placement). The previous works in [2, $7,10]$ reveal either analytically or through simulations that the MSE is proportional to the standard deviation of TDOA estimates, and it increases exponentially with the increase of the tracking range outside the baseline while it becomes approximately independent of the tracking range once if the target is inside the baseline.

In order to analyze the tracking error "behavior" and gain some insight regarding achievable tracking resolution in a 3D space, several Matlab simulations were performed using the TDOA tracking method described in Section II. The results of these simulations are discussed below.

\section{A. Baseline Configuration and Target Trajectory}

Given a tracking area, it is found that the placement of the receivers has significant impact on the tracking performance [11]. Intuitively, the optimum receiver configuration is to distribute five receivers uniformly in the given space. By taking into account of the feasibility of receiver placement, an "optimum" configuration called "Twisted Square Configuration" is identified in [11]. As shown in Figure 1, this configuration places one receiver at the origin of the given space, and places four receivers at the vertices of a "Twisted Square" (two receivers in the $x-z$ plane and two receivers in the $y-z$ plane). The distance from the vertex to the origin is defined as the radius of the configuration baseline.

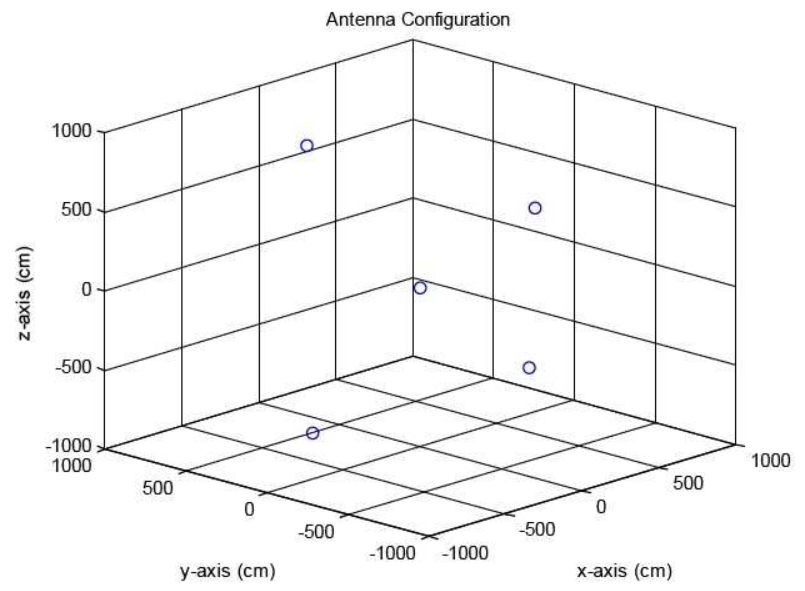

Fig. 1 Twisted Square Configuration of Receivers

The "proximity tracking" is defined as a tracking scenario that the target is inside the baseline, which means the tracking range is not greater than the radius of the 
baseline. In order to evaluate the average tracking performance at any arbitrary range, the target travels over the surface of a $3 \mathrm{D}$ sphere with the radius of the tracking range. Figure 2 shows the target trajectory which forms a $3 \mathrm{D}$ sphere with radius 20 feet. The same trajectory runs for the different settings. The default simulation setting includes: standard deviation of TDOA estimates $10 \mathrm{ps,}$ tracking range 20 feet, and radius of baseline 20 feet.

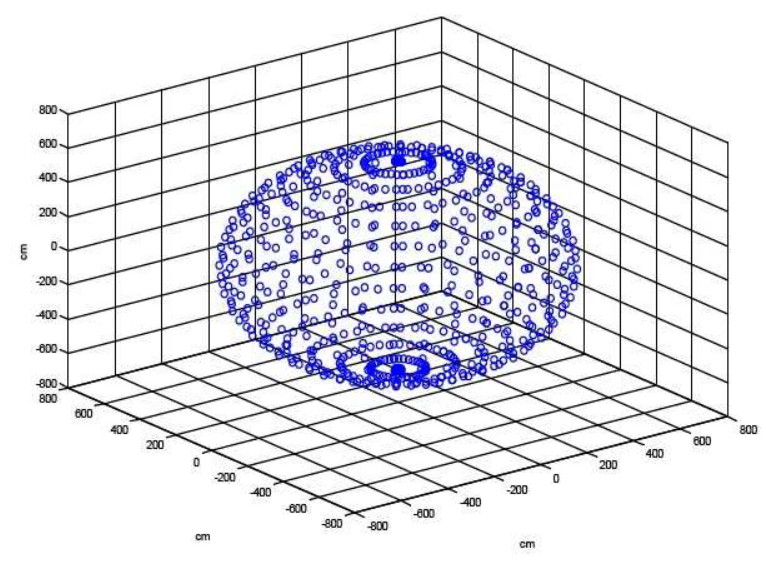

Fig. 2 Sphere formed by the target 3D trajectory

\section{B. Tracking error vs. TDOA estimates noise level}

First, the relationship between the tracking error (MSE) and the TDOA estimate noise level has been studied for noisy TDOA estimates. In Figure 3, the simulation results show that the tracking error is proportional to the standard deviation of TDOA estimates. If the required tracking accuracy is at the center meter level, the TDOA estimate noise level should be controlled below $10 \mathrm{ps}$.

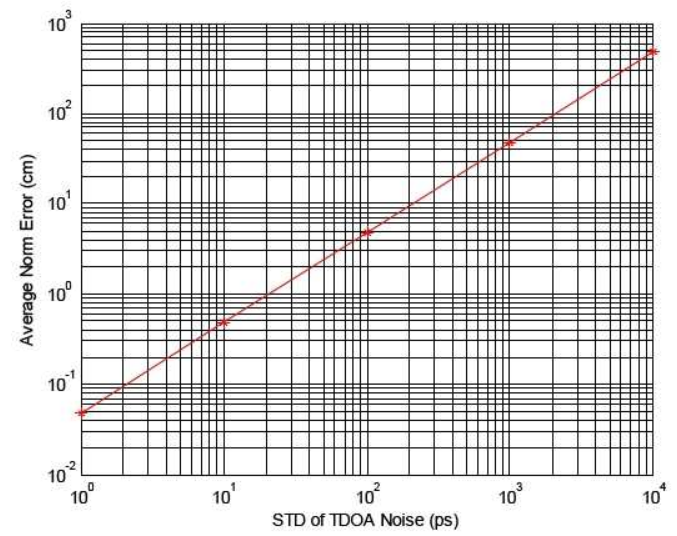

Fig. 3 Tracking Error vs. TDOA Noise Level

\section{Tracking error vs. tracking range}

Second, the relationship between the tracking error (MSE) and the tracking range has been studied. In Figure 4, the simulation results show that the tracking error increases exponentially with the increase of the tracking range when the target is outside the baseline (Note: the default radius of baseline is 20 feet, about $610 \mathrm{~cm}$ ). The interesting observation is that if the target is inside the baseline, the tracking error is approximately independent of tracking range and the tracking error inside baseline is well maintained below $0.5 \mathrm{~cm}$. This motivates the next step of investigation on whether this behavior holds when the radius of baseline changes.

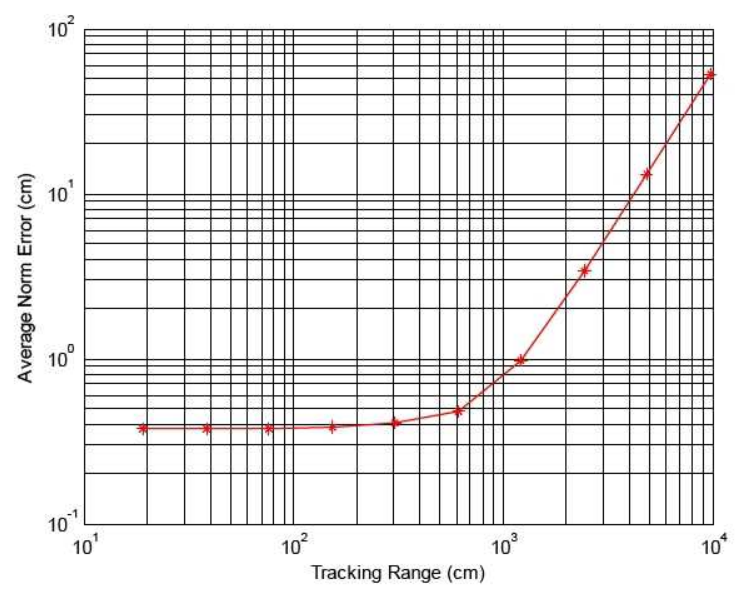

Fig. 4 Tracking Error vs. Tracking Range

\section{Tracking error vs. radius of baseline}

Third, the relationship between the tracking error (MSE) and the radius of baseline has been studied. In Figure 5, the simulation results show that the tracking error does not change as the baseline size changes. As the radius of baseline is changed from a few feet to a few miles, the tracking error maintains below $0.5 \mathrm{~cm}$. This is encouraging and it shows that the fine tracking precision can be achieved at any given range if it is feasible to extend the radius of the baseline to that range while maintain the low TDOA estimate noise level.

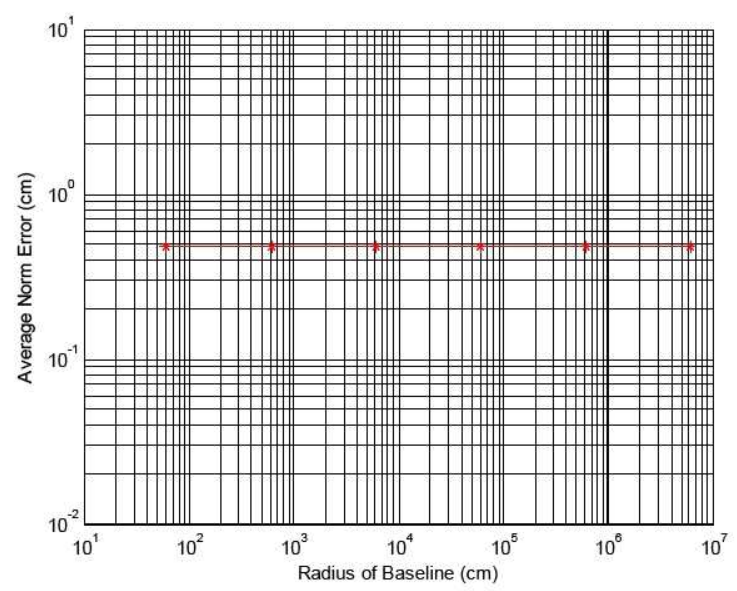

Fig. 5 Tracking Error vs. Radius of Baseline 
The above simulation results show that the tracking performance has the same "behaviors" in a 3D space as in a 2D space if the receiver baseline is properly configured. The tracking performance can be further improved by increasing the baseline size if it is feasible and by decreasing the TDOA noise level.

\section{SYSTEM DESIGN AND FIELD TEST}

\section{A. System Design}

A prototype system was designed using the UWB radios PulsON 210 from Time Domain Corporation. The UWB impulse radio (UWB-IR) technology is exploited in the design and implementation of the prototype location and tracking system due to its capacity for fine time resolution, low power spectral density, resistance to multipath interference. A key element of the system design philosophy was to avoid introduction of system components or structure that would in any way degrade the fine time resolution of the UWB signal since it is critical for precise tracking. In keeping with this goal, a "One-Receiver-Five-Antenna" design was adopted in order to avoid the degradation in time resolution introduced by synchronization errors among receivers. A tracking prototype system was designed, which connects five antennas through a power combiner to one UWB receiver using low-loss, phase aligned interconnect cables with precisely calibrated delays. In this way, five delayed versions of the received UWB pulse are obtained at the single receiver.

To estimate the time delay between pulses, a method called Cross-Correlation plus Peak Detection (CCPD) has been introduced. Cable delays were chosen such that the five delayed versions of the signal from different antennas at the receiver fit within a scanning window 200 nanoseconds in duration. A peak detector is first used to detect the direct-path arrival of each signal and separate it from possible multipath arrivals. Cross correlation between the two direct-path arrivals is then employed to estimate the precise time delay between the signals. The maximum cross-correlation coefficient between any two of the arriving signals gives the optimal (maximumlikelihood) estimate of the time delay between the two. The TDOA estimates are fed into the TSWLS algorithm described in Section II and the transmitter position is computed and displayed in the tracking window.

The configuration of the UWB TDOA High Resolution 3D Proximity Tracking System is shown in Figure 6. On the transmitting side, one UWB radio with an omniantenna is mounted on the top of the target; on the receiving side, a one-radio-five-antenna configuration is chosen to eliminate the synchronization issue among receivers. Five receiving antennas are connected to one UWB radio through a power combiner. The optimum antenna location configuration "Twisted Square" is chosen based on the study results descript in [11]. One antenna is set up at the origin of the coordinates system while other four antennas are located on the circle of a predefined radius. This configuration places two antennas in the $x-z$ plane and two antennas in the $y-z$ plane.

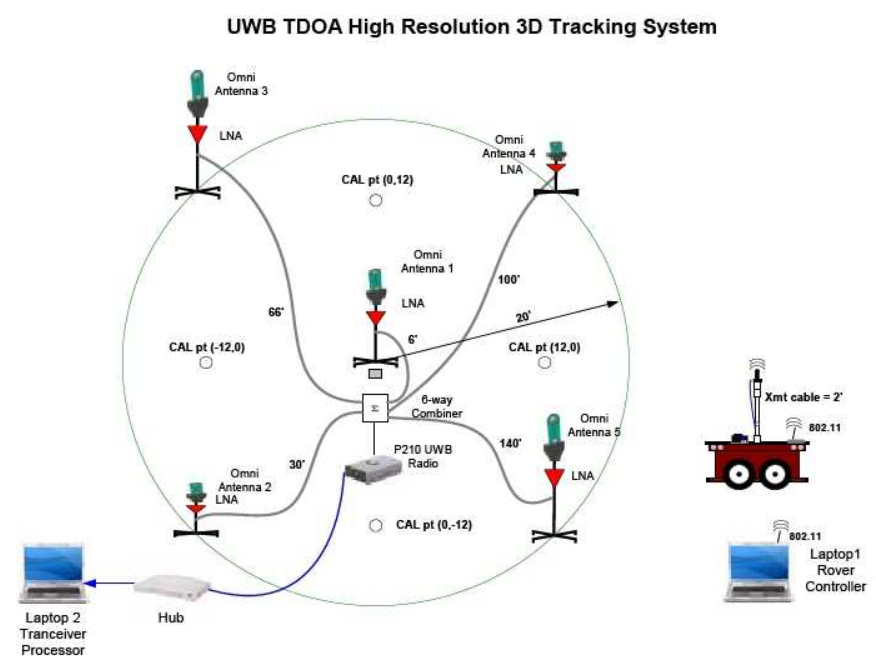

\section{Fig. 6 UWB TDOA 3D Proximity Tracking System Configuration}

\section{B. Field Test}

The field test of the UWB TDOA High Resolution 3D Proximity Tracking System was conducted at Honeywell's "Moonyard" robotic control test facility in Greendale, Arizona. The tests setup is shown in Figure 7. One receiving antenna is located at the "origin" of the "Moonyard" while other four are located on a circle of radius 20 feet. The center antenna is set at height of 4 feet. Among other four antennas, two are set low at a height of 2 feet while two are set high at a height of 6 feet. The span of Z-dimension is about 4 feet. Figure 8 shows the target is climbing up to a mound that is one terrestrial feature of this 3D "Moonyard".

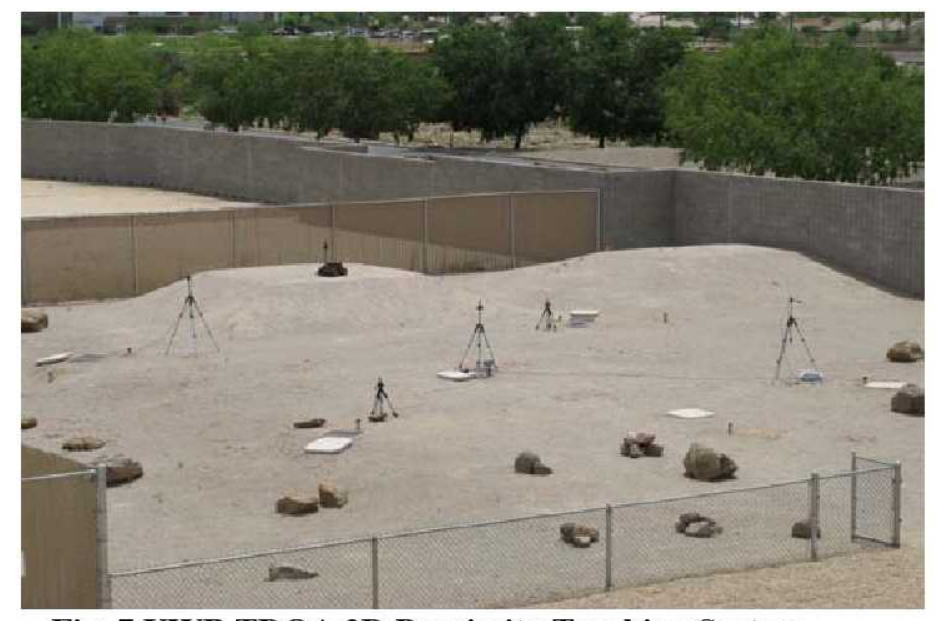

Fig. 7 UWB TDOA 3D Proximity Tracking System Setup at "Moonyard" 


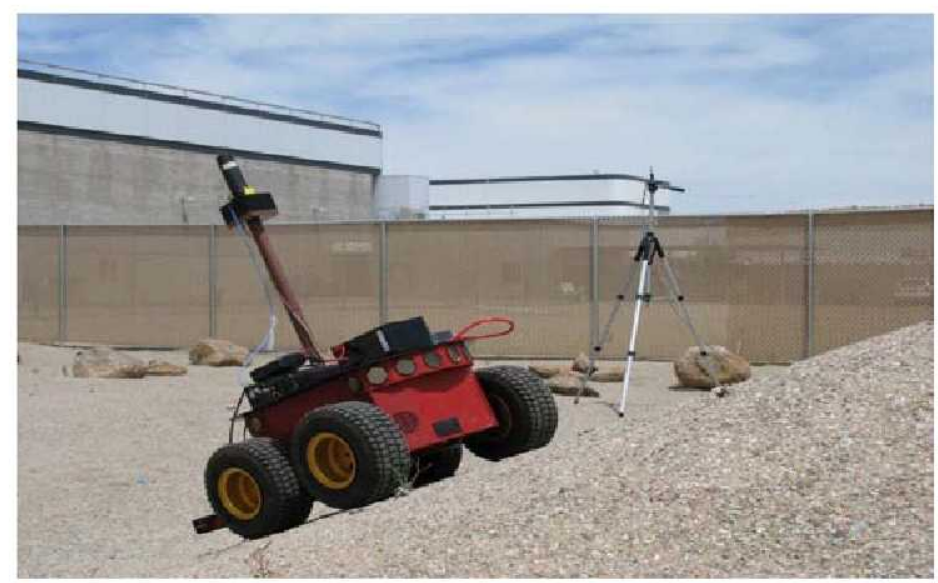

Fig. 8 Target Tracked by UWB 3D Tracking System

A reference tag is used to calibrate the system to obtain the cable delays. Before operating the tracking system, a transmitter is placed at a known position to act as a reference tag and the cable delays are computed based on the multiple arrivals from the reference transmitter position. Once the cable delays are known, the CCPD finds the time delays between signals. The TDOA estimates can be obtained at the accuracy of about ten picoseconds. The TDOA data are fed into the TSWLS algorithm and the transmitter position is calculated as the output and displayed in the tracking window of the graphical user interface (GUI).

The UWB signals received through five receiving antennas from the transmitting antenna on the target are shown in Figure 9. The tracking algorithm utilizes the TDOA information generated from the receiving signal to determine the location of the target. The tracking accuracy is tested. The test data shows that the average tracking accuracy is less than one inch in X-axis and Yaxis and is about two inches in Z-axis. It is conjectured the tracking accuracy degradation in Z-axis is due to the limited span of Z-dimension. Real time trajectory runs are also tested. One of the trajectories in X_Y Plane recorded by the UWB tracking system GUI is shown in Figure 10. The update rate of the tracking data is about $1 \mathrm{~Hz}$.

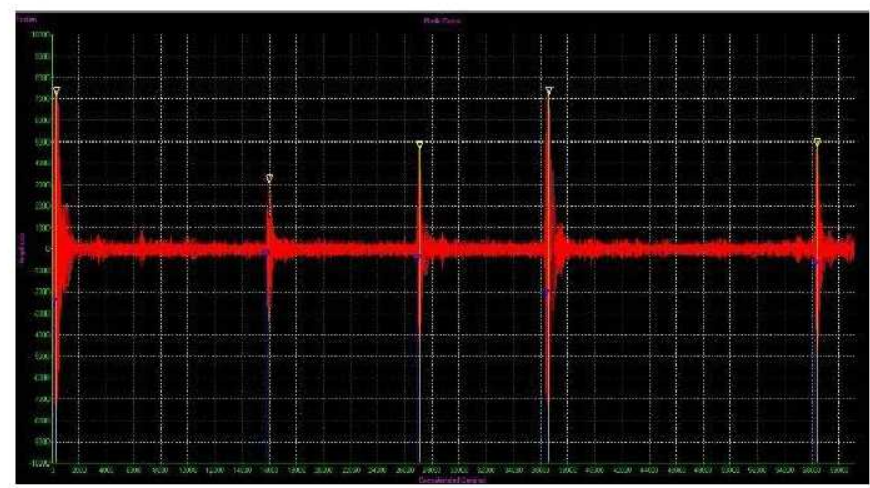

Fig. 9 UWB Signals Received through Five Receiving Antennas

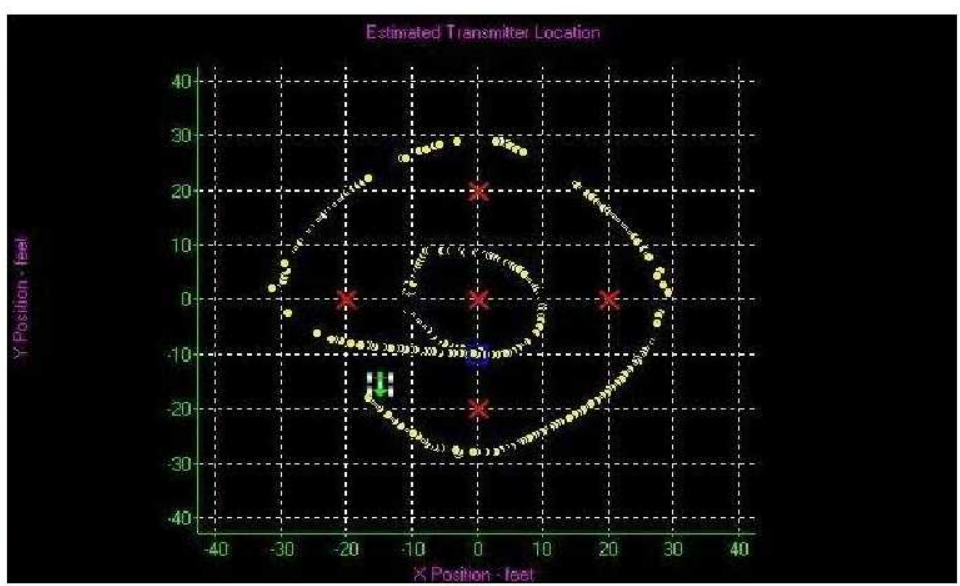

Fig. 10 Tracked Trajectory of the Target in X_Y Plane

\section{Performance Investigation}

In order to evaluate the impact of the Z-dimension compression on the tracking performance, two scenarios are simulated and compared. The simulation configuration matches the set up of the field test of the UWB TDOA High Resolution 3D Proximity Tracking System at Honeywell's "Moonyard" robotic control test facility in Greendale, Arizona. One scenario is the default antenna setting, in which each dimension $(\mathrm{X}, \mathrm{Y}, \mathrm{Z})$ has the same span of 40 feet; the other one is the test antenna setting as discussed above, the span of Z-dimension is compressed to 4 feet.

First, the tracking norm error is evaluated through simulation. The simulation results show that the average norm error for the default setting is about $0.5 \mathrm{~cm}$ and the average norm error for the test setting is about $0.8 \mathrm{~cm}$. This indicates that the compression of Z-dimension causes the increase of the tracking norm error.

Furthermore, the tracking error in each dimension is investigated through simulation. Figure 11 shows the tracking error analysis data in three dimensions for the default setting while the tracking error analysis data in three dimensions for the test setting is shown in Figure 12. The simulation results show that the average error in $\mathrm{X}$-dimension and $\mathrm{Y}$-dimension is about $0.3 \mathrm{~cm}$ and the average error in Z-dimension is about $0.2 \mathrm{~cm}$; when the $\mathrm{Z}$-dimension is compressed, the average error in Zdimension increases to about $0.6 \mathrm{~cm}$ while the average error in X-dimension and Y-dimension still maintains at the same level about $0.3 \mathrm{~cm}$. This analysis data explains why Z-dimension error is about twice as the error in Xdimension and Y-dimension for test data. It also evidently demonstrates that the tracking accuracy degradation in Zaxis is due to the limited span of Z-dimension. 


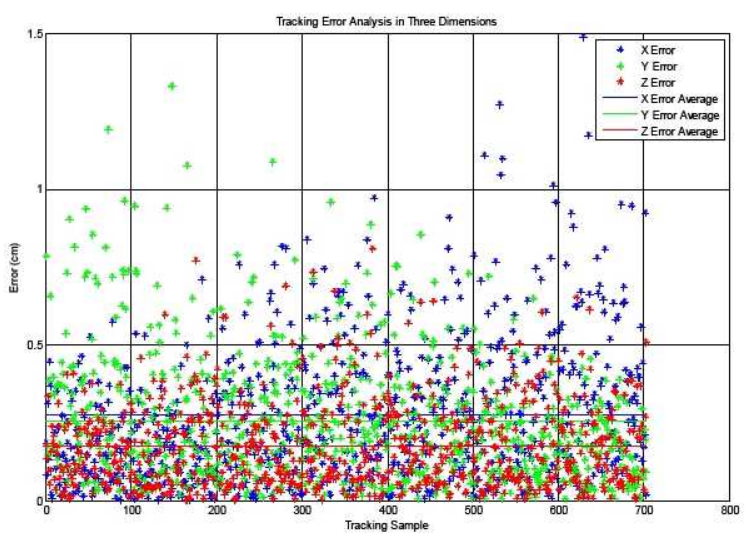

Fig. 11 Error in three dimensions for default setting

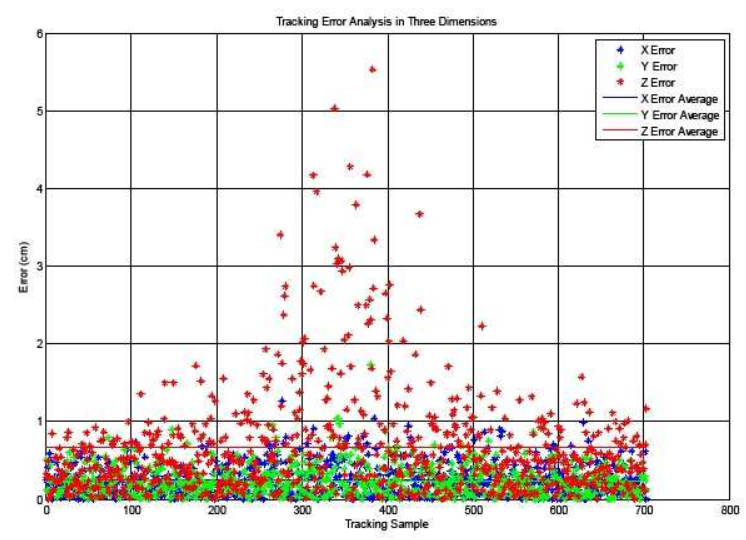

Fig. 12 Error in three dimensions for test setting

\section{CONCLUSION}

A prototype UWB TDOA High Resolution 3D Proximity Tracking System has been designed, implemented, tested, and proven feasible for providing positioning-awareness information in a 3D space to a robotic control system. UWB technology has been exploited to implement the tracking system due to its properties such as fine time resolution and low power spectral density. The TDOA tracking method has been employed to avoid synchronization problems between the transmitter and the receiver. Furthermore, the "One-Receiver-Five-Antenna" design eliminates the synchronization issue among receivers. Simulations show that the desired fine tracking resolution can be achieved for any size of proximity tracking if the extension of the baseline radius is feasible. Filed tests demonstrate that this UWB tracking system can track the moving target in real time and an inch-level tracking accuracy can be achieved. Future work includes developing a scheme to enlarge the span of Z-dimension for Z-axis tracking data accuracy improvement, and expanding the tracking area through automatic gain control and using advanced synchronization techniques.

\section{REFERENCES}

[1] J. Ni, et al; "Ultra-Wideband Time-Difference-OfArrival Two-Point-Tracking System", NASA JSC Internal Tech Report, March 2009.

[2] J. Ni, et al; "UWB TDOA Proximity High Resolution Tracking System", IEEE Galveston Bay Section Symposium for Space Applications of Wireless \& RFID 2007, Houston, TX, May 8-9, 2007.

[3] J. Ni, et al; "UWB Tracking Algorithms - AOA and TDOA", AIAA Houston Annual Technical Symposium 2006, Houston, TX, May 19, 2006.

[4] J. Ni, et al; "Ultra- Wideband Two-Cluster Tracking System Design with Angle of Arrival Algorithm", IEEE Radar Conference 2006, Verona, NY, April 24-27, 2006.

[5] J. Ni, et al; "UWB Tracking System Design for Lunar/Mars Exploration", First IEEE International Conference on Wireless Broadband and Ultra Wideband Communications AuS Wireless 2006, Sydney, Australia, March 13-16, 2006.

[6] J. Ni, et al; "Design and Performance Analysis of a UWB Tracking System for Short Range Space Applications", AIAA Houston Annual Technical Symposium 2005, Houston, TX, May 6, 2005.

[7] J. Ni, et al; "Design and Performance Analysis of a UWB Tracking System for Space Applications", 2005 IEEE/ACES International Conference on Wireless Communications and Applied Computational Electromagnetics, Honolulu, Hawaii, April 3-7, 2005.

[8] J. Ni, et al; "UWB Tracking System Design for FreeFlyers", AIAA Space 2004 Conference and Exposition, San Diego, CA, September 28-30, 2004.

[9] Chan, Y.T.; Ho, K.C.; "An efficient closed-form localization solution from time difference of arrival measurements", Acoustics, Speech, and Signal Processing, 1994. ICASSP-94., 1994 IEEE International Conference on ,Volume: ii , 19-22 April 1994 Pages:II/393 - II/396 vol.2

[10] Richard J. Barton; "Design and Performance Evaluation of a UWB Communication and Tracking System for Mini-AERCam", NASA Faculty Fellowship Program 2004 final report.

[11] J. Ni, et al; "Ultra-Wideband Time-Difference-OfArrival Three-Dementional Tracking Algorithm and Optimum Configuration", JSC/NASA internal report, November 2008.

[12] Time Domain Corporation, "PulsON Technology Overview",

http://www.timedomain.com/Files/downloads/techpapers/ PulsONOverview7 01.pdf 